Research Report

\title{
The hernia sac-A suitable source for obtaining mesenchymal stem cells猔它动
}

\author{
Alpha Dian-Yu Lin, MD ${ }^{\mathrm{a}, \mathrm{b}, \mathrm{c}}$, Min-Che Tung, MD ${ }^{\mathrm{b}, *}$, Chin-Heng Lu, MD ${ }^{\mathrm{b}}$ \\ a Joshua Taipei Hernia Center, Central Clinic E' Hospital, Taipei, Taiwan \\ b Division of Urology, Department of Surgery, Tungs' Taichung MetroHarbor Hospital, Taichung, Taiwan \\ c Department of Urology, College of Medicine and Shu-Tien Urological Research Center, National Yang Ming Chiao Tung University, Taipei, Taiwan
}

\section{A R T I C L E I N F O}

\section{Article history:}

Received 12 May 2021

Received in revised form 29 July 2021

Accepted 17 August 2021

Available online 28 August 2021

\section{Keywords:}

mesenchymal stem cell

inguinal hemia

hemia sac

\begin{abstract}
A B S T R A C T
Background: Inguinal hernia sac, extended tissue from peritoneum, gradually enlarged in size with hernia disease time and prolapsed tissue volume. We hypothesize that mesenchymal stem cells are present in the development of hernia sac. The current study aimed to test the hypothesis that hernia sac, which is often resected and discarded as medical waste, contains mesenchymal stem cells and thus might be a suitable source to harvest mesenchymal stem cells.

Methods: Between July 2019 and June 2020, 4 hernia sacs were resected during hernia surgery and then obtained for mesenchymal extraction using the Miltenyi gentleMACS Dissociator. The presence of mesenchymal stem cells was determined by the markers CD105, CD73, and CD90, with assessment of the expressions $\geq 95 \%$, whereas markers CD45, CD34, CD11b, CD19, and HLA-DR were used to assess lack expression ( $\leq 2 \%)$. Moreover, von Kossa staining, Alcian blue staining, and Oil Red O staining were used to verify the cells' ability for differentiation. Results: Cells retrieved from the hernia sacs displayed a spindle-shaped morphology and exhibited adherence to plastics. The cell surface immunophenotypic profile was confirmed using surface markers APC-A (CD73), FITC-A (CD90), and PerCP-Cy5-5-A (CD105), with results showing 100\%, 100\%, and 99.2\%, respectively, strongly indicating the presence of mesenchymal stem cells. Moreover, staining of in vitro cell cultures showed in vitro differentiation of precursor cells into osteoblasts, adipocytes, and chondroblasts, suggesting positive differentiation ability and identification of mesenchymal stem cells.

Conclusion: Inguinal hernia sac is a novel source of mesenchymal stem cells that can be easily obtained and stored for future usage.
\end{abstract}

(C) 2021 Published by Elsevier Inc. This is an open access article under the CC BY-NC-ND license (http://creativecommons.org/licenses/by-nc-nd/4.0/).

\section{INTRODUCTION}

Mesenchymal stem cells (MSCs), defined as multipotent stromal cells that can mature or differentiate into various cell types, have a myriad of clinical applications in tissue engineering, regenerative surgery, MSC-rich tissue scaffolding, etc. These cells can be found in a variety of places throughout the body, such as the bone marrow, umbilical cord tissue, adipose tissue, developing tooth bud of the mandibular third molar, and amniotic fluid [1-5]. The most common source for obtaining MSCs postnatally has been from the umbilical cord, including umbilical cord blood cells, amnion/placenta, umbilical cord vein, or umbilical cord matrix cells [6]. Among adults, however, MSCs are commonly derived from the bone marrow [3]. Other sources include adipose tissues,

is Source of funding: N/A

项败 Whether the paper is based on a previous communication to a society or meeting: N/A

* Corresponding author at: Tungs' Taichung Metro Harbor Hospital, Taiwan, No. 699, Sec. 8, Taiwan Blvd., Taichung City 435, Taiwan.

E-mail address: tungminche@gmail.com (M.-C. Tung). which could be a much easier and more inexpensive source compared to the bone marrow [7]. However, several other human body parts have been found to contain mature MSCs, with a recent study showing the peritoneum as one such source for MSCs. Peritoneal MSCs can be separated from peritoneal or peritoneal dialysis fluid [8,9]. Hernia sacs originate from peritoneum and gradually downward developed in size with hernia disease time and prolapsed tissue volume. The sac is often respected during hernia surgery procedures. It is our hypothesis that hernia sac contains MSCs as to develop the gradually larger pockets to contain and hang up the heavier and heavier weight of prolapsed herniated tissues in hernia sac.

If the hypothesis is proven, patients with inguinal hernia could have one more choice to store their MSCs. Instead of discarding the hernia sac as medical waste, surgeons could preserve the excised hernia sac without additional surgical procedures. MSCs from hernia sacs are easier and more practical to obtain compared to current common sources, such as umbilical cord tissue or bone marrow $[10,11]$. This study aimed to confirm whether MSCs can be harvested from hernia sacs discarded following hernia surgery. 

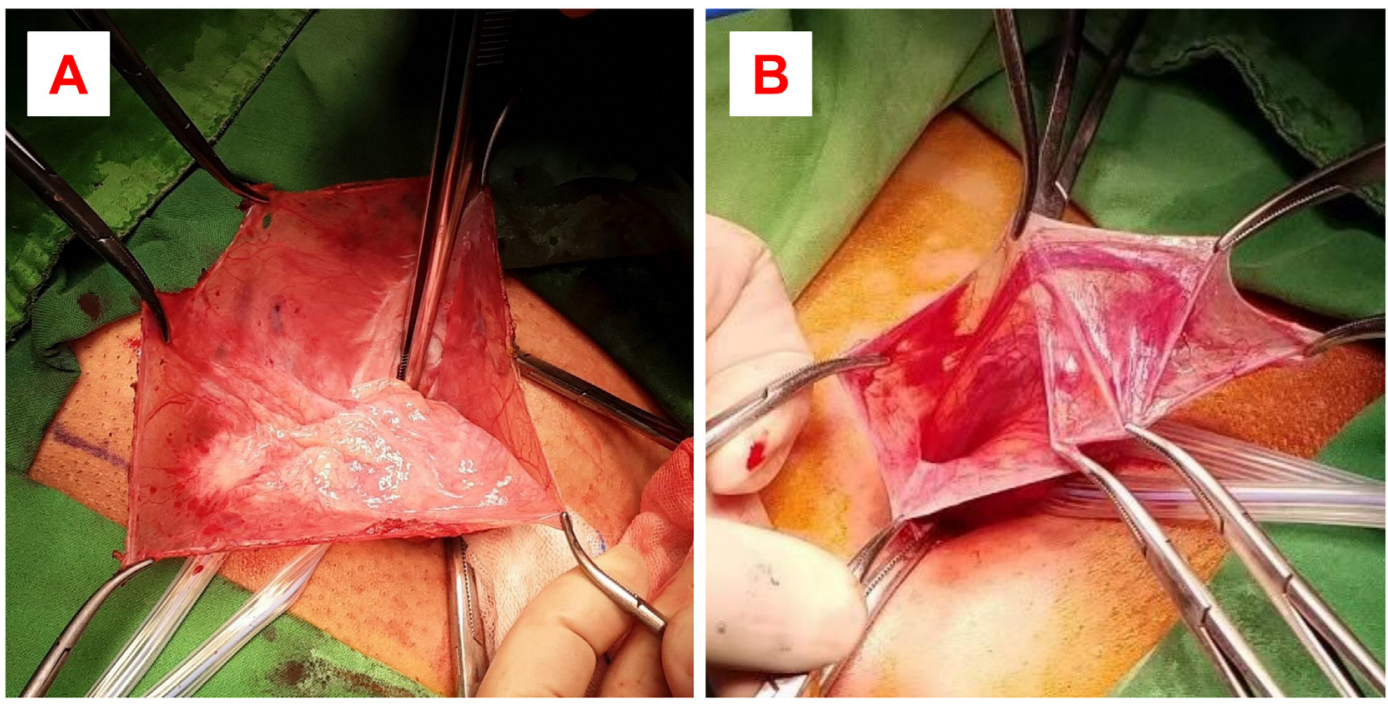

Fig 1. Hernia sac and inguinal hernia.

\section{MATERIALS AND METHODS}

Cell Dissociation From Hernia Sacs. Surgery for adult inguinal hernia (Fig 1) involves performing the open method with inguinal incision. Fresh hernia sacs obtained from 4 randomly selected inguinal hernia surgeries were harvested and immediately sent to the laboratory. Details regarding these 4 patients are summarized in Table 1. MSCs were harvested within $48 \mathrm{~h}$. Peritoneal tissue from hernia sac was first isolated and cut into small pieces, after which the mesenchymal cells within them were obtained using the Miltenyi gentleMACS Dissociator, which has been used for the semiautomated dissociation of tissues into single-cell suspensions or thorough homogenates (www.miltenyibiotec.com). The dissociated suspension was filtered through 40- $\mu \mathrm{m}$ strainer (BD Falcon) and cultured in alpha-Minimal Essential Medium (Thermofisher) plus 10\% fetal bovine serum (Hyclone) at $37^{\circ} \mathrm{C}, 5 \% \mathrm{CO} 2$ and humidified atmosphere. This study had been reviewed and approved by institutional review board of Tung's Taichung Metro Harbor Hospital. All patients/participants provided written informed consent prior to participation (IRB \#109060).

Cell Identification and Morphology. The presence of MSCs was determined by using the following minimum criteria for defining multipotent MSCs according to The International Society for Cellular Therapy [12]: (1) adherence to plastic, (2) specific surface antigen (Ag) expression, and (3) multipotent differentiation potential.

MSCs were placed in plastic culture plates to observe their morphology and ability for plastic adherence when maintained in standard culture conditions using tissue culture flasks. The hematopoietic cells, mature adipocytes, etc, would not attach to the plastic plate in this condition. Positive markers selected for our studies were based on existing literature and antibody availability for human MSCs [12]. The markers CD105, CD73, and CD90 were used to assess expressions $\geq 95 \%$, whereas markers CD45, CD34, CD11b, CD19, and HLA-DR were used to assess lack of expression ( $\leq 2 \%)$. All positive CD markers used (CD105, CD73, and CD90) had an expression of $\geq 95 \%$ when tested.

Cell Morphology and Differentiation. When MSCs grew to $90 \%$ confluent, the medium was shifted to differentiation medium for the assay of trilineage capability. The differentiation media were StemMACS Osteodiff medium, StemMACS Chondrodiff medium, or StemMACS Adipodiff medium, respectively (Miltenyi Biotec). After 3 weeks, cells underwent von Kossa staining to verify osteogenesis, Alcian blue stain to verify ability to undergo chondroblasts, and Oil Red O staining to determine adipogenesis.

\section{RESULTS}

This study first assessed the morphology of the cells retrieved from hernia sacs obtained following inguinal hernia surgeries and their ability to adhere to plastics. All cells from the 4 patients displayed a spindle-shaped morphology and exhibited adherence to plastics (Fig 2), partly indicating the presence of MSCs [13].

Determining Reliable Positive Markers for Mesenchymal Stem Cell Identification. The cell surface immunophenotypic profile, along with the following positive CD markers, was determined: CD73 (100\%), CD90 (100\%), and CD105 (99.2\%). The following negative CD markers were also assessed to further verify the presence of MSCs: CD11b, CD19, CD34, CD45, and HLA-DR (Fig 3). Accordingly, all negative CD markers tested (CD11b, CD19, CD34, CD45, and HLA-DR) had an expression of $\leq 2 \%$ (Fig 3), strongly suggesting the presence of MSCs.

Differentiation Ability of Cells. The in vitro differentiation of precursor cells into osteoblasts, adipocytes, and chondroblasts was determined through staining of in vitro cell cultures. Von Kossa staining confirmed

Table 1

Summary of patients' characteristics.

\begin{tabular}{|c|c|c|c|c|}
\hline & Case 1 & Case 2 & Case 3 & Case 4 \\
\hline Age & 54 & 36 & 71 & 42 \\
\hline Sex & Male & Male & Male & Male \\
\hline Height & 165 & 166 & 156 & 174 \\
\hline Weight & 70 & 77 & 56.2 & 87 \\
\hline Smoking & No & No & No & No \\
\hline Hernia size & $32 \times 14.5 \times 2.5 \mathrm{~cm}$ & $6 \times 4 \times 8 \mathrm{~cm}$ & $2 \times 3 \times 5 \mathrm{~cm}$ & $3 \times 3 \times 5 \mathrm{~cm}$ \\
\hline Medical history & Hepatitis B, hypertension & No & Prostate cancer & Lung arteriovenous aneurysm, scrotal spermatic varicocele \\
\hline Surgical history & No & No & Robot-assisted laparoscopic radical prostatectomy & Embolization \\
\hline
\end{tabular}



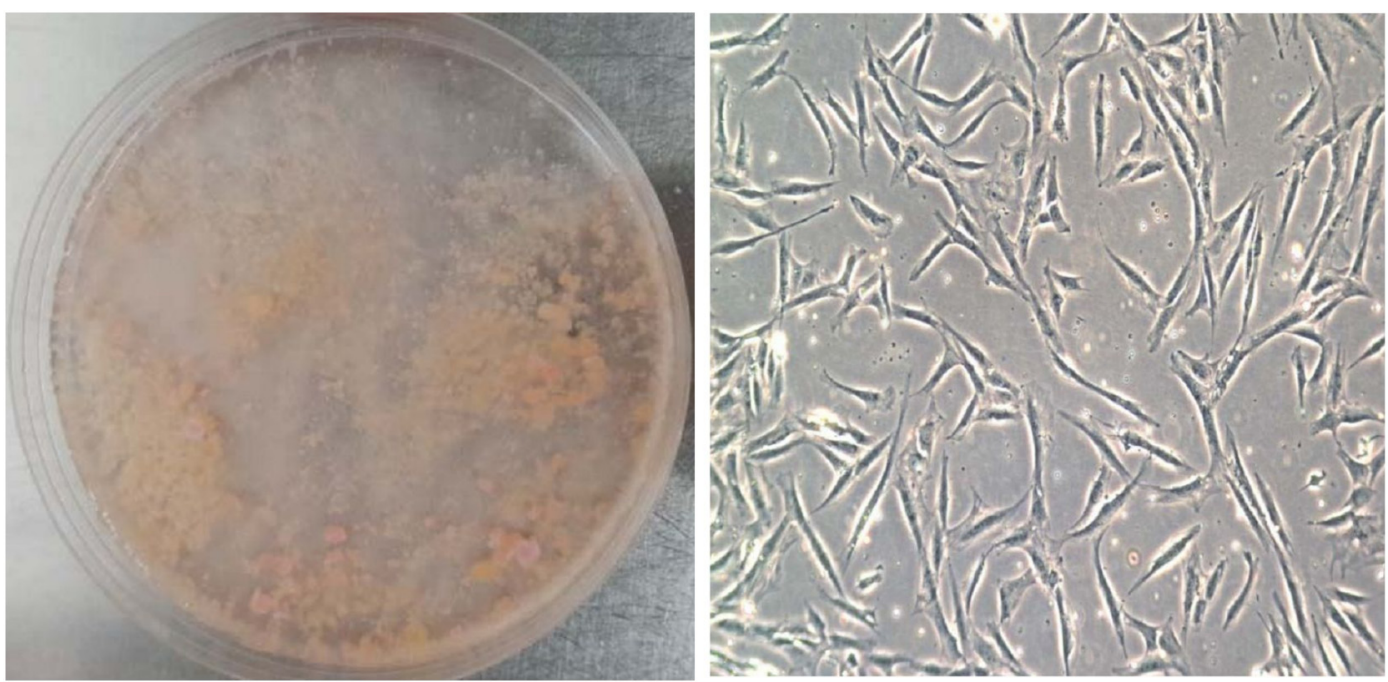

Fig 2. Plastic adherence properties and morphology of cultured hernia sac-derived cells.

differentiation into osteoblasts, Oil Red O staining demonstrated differentiation into adipocytes, and Alcian blue staining confirmed differentiation into chondroblasts [12]. Figure 4 revealed positive differentiation ability and identification of MSCs following von Kossa, Alcian blue, and Oil Red O staining (Fig 4). The uninduced cells showed no sign of differentiation.

\section{DISCUSSION}

The current study demonstrated, for the first time, that hernia sacs removed during inguinal hernia surgeries can be a good source for
MSCs in terms of both quality and quantity. The morphology of the cultured cells (Fig 2), as well as the different types of staining used to identify the cells' ability to differentiate, was indicative of MSCs. As observed in the images following von Kossa, Oil Red O, and Alcian blue staining, all 3 were indicative of adipogenesis, chondrogenesis, and osteogenesis, respectively (Fig 4). This further confirmed that MSCs are abundantly present in adipose tissue derived from hernia sacs obtained following inguinal hernia repair surgeries [14].

The most common source for MSCs postnatally has been from the umbilical cord, which includes the umbilical cord vein, blood cells, amnion/placenta, or matrix cells [6]. In the absence of umbilical cord stem
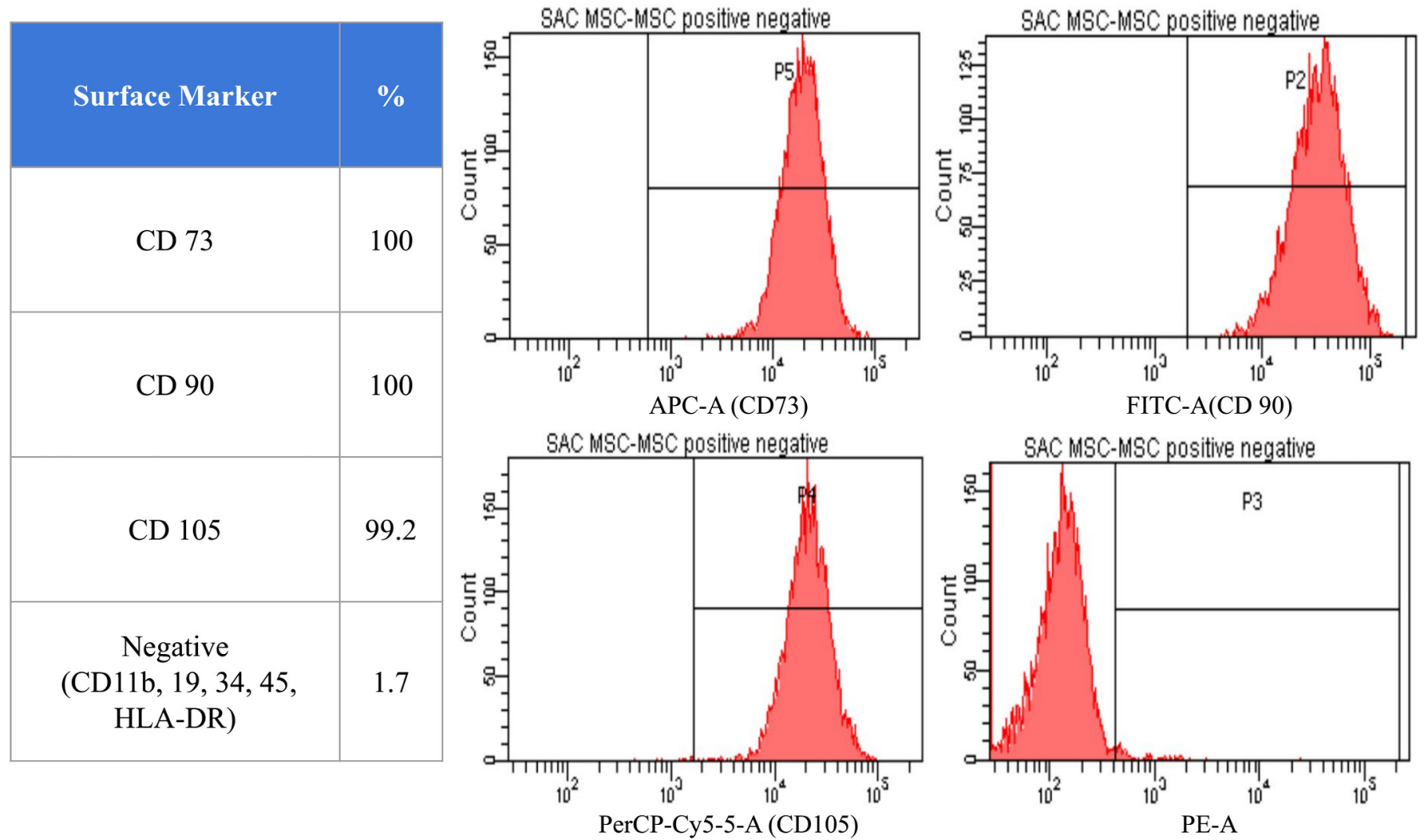

Fig 3. Positive and negative markers used to identify the presence of mesenchymal stem cells in the hernia sac. 


\section{Differentiation Ability}

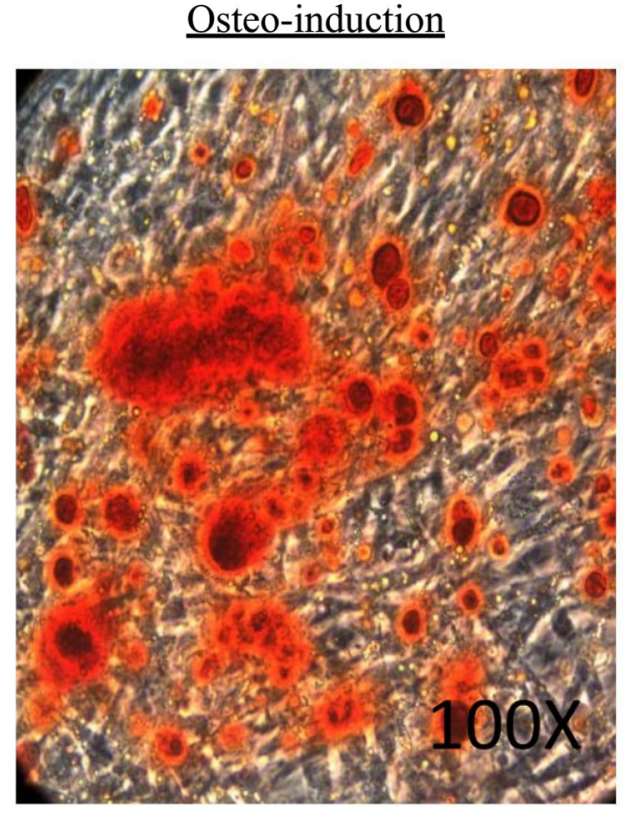

Osteogenesis: van Kosa staining $\underline{\text { Alcian blue staining }}$

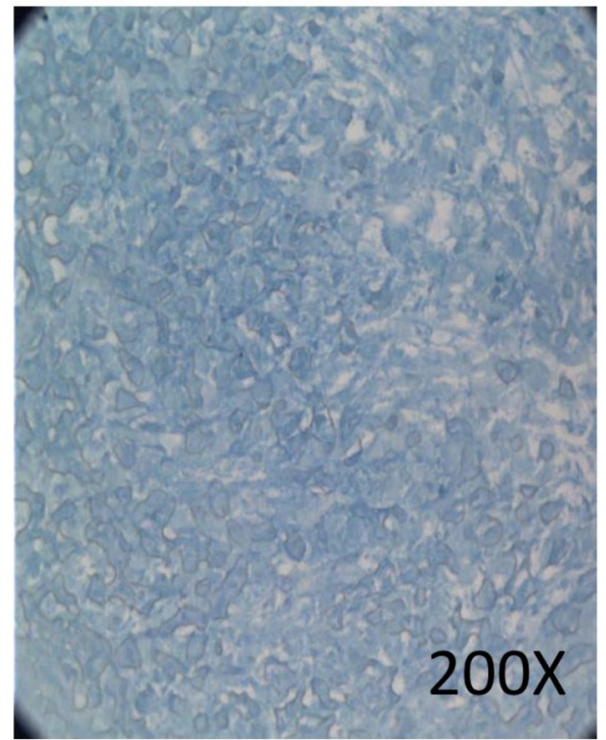

Chondrogenesis: Alcian blue staining $\underline{\text { Adipo-induction }}$

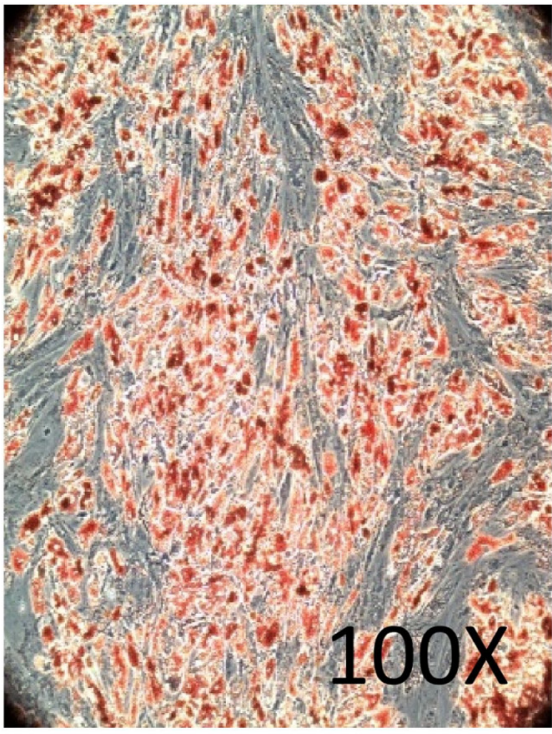

Adipogenesis: Oil red $\mathrm{O}$
staining

Fig 4. Differentiation ability and identification of mesenchymal stem cells following von Kossa, Alcian blue, and Oil Red O staining.

cells after birth, the bone marrow can be another common source of MSCs. However, bone marrow MSCs are particularly difficult to harvest in most patients and require invasive procedures. The present study confirmed that hernia sacs could provide enough volume of mesenchymal tissues with sufficient quality. In the current era, short- or longterm storage facilities for hernia sacs removed following surgery are widely available worldwide. As such, these MSCs could be harvested and stored for further or additional practical use (eg, tissue engineering; tissue expansion; MSC-rich tissue scaffolding; and MSC cultures for functional tissues, such as insulin-secreting tissues, cartilage, and bone). Therefore, rather than discarding the entire hernia sac as a medical waste, it could be harvested and stored for future isolation of MSCs to be used for replacing and restoring damaged or dysfunctional cells. MSCs could also be engineered to deliver therapeutic proteins.

The current study suggested the presence of MSCs in the hernia sac excised following surgery, which can be used for a variety of important tasks, such as regenerative medicine and disease therapeutics [15-17]. Some aged patients who develop a hernia present with degenerative diseases, such as osteoarthritis of knee or coronary heart diseases, which may benefit from MSC treatment [18]. As such, future studies aimed at treating these degenerative diseases using MSCs harvested from the autologous hernia sac are warranted. MSCs can also be easily isolated during a variety of surgical procedures. These approaches are safer and can obtain larger amounts of stem cells compared to that used for extracting bone marrow MSCs. One such source is adipose tissue removed during liposuction and resected fat, which are feasible sources of MSCs for regenerative medicine $[19,20]$. However, given that adipose tissue can also be found in the inguinal region of the body, new developments and observations indicate that hernia sacs removed during inguinal hernia repair surgeries can be a good source of MSCs.

Although other stem cells and bone marrow MSCs have many similar biological characteristics, some differences in their immunophenotype, differentiation potential, transcriptome, proteome, and immunomodulatory activity have been identified [10]. Some of these differences may represent specific features of bone marrow MSCs and other stem cells, whereas others are suggestive of the inherent heterogeneity [21]. Different isolation and culture protocols may induce different characteristics. MSCs displayed certain enhanced functionalities when derived from specific places [13]. Most importantly, however, despite the minor differences between these MSC populations, stem cells from some other sources seem to be as effective as those derived from the bone marrow for clinical application and may even promote better outcomes in certain applications [22-24]. Further studies are needed to explore the clinical application of MSC obtained from hernia sacs and compare their efficacy with MSCs from other sources. There are different methods of inguinal hernia surgery; basically, there are 2 routes to approach: open method and laparoscopic method. The current study applied open hernia surgery to harvest the hernia sac without additional procedures. There is no increase in surgical risk, operation time, or anesthesia loading. Different from throwing the waste, aseptic preservation should be alerted in the scrubbing table. For more extensive applications in the future, it is necessary to investigate further the harvesting of hernia sac in laparoscopic hernia surgery.

In conclusion, our study confirmed that hernia sacs could provide sufficient quality and quantity of MSCs, indicating that the hernia sac could be a promising source [1-24] of MSCs for further tissue engineering, regenerative surgeries, and further possible applications in patients with hernia.

\section{Acknowledgments}

AD: collection and assembly of data, data interpretation, manuscript writing, and final approval of the manuscript. MC: data analysis and interpretation, final approval of the manuscript. $\mathrm{CH}$ : data analysis and interpretation, manuscript editing, manuscript revision, and final approval of manuscript. All authors contributed to the article and approved the submitted version.

\section{Disclosure}

The authors have no disclosures and competing interests. 


\section{Conflict of Interest}

The authors declare no commercial or financial relationships that could be construed as a potential conflict of interest.

\section{Credit Author Statement}

Alpha Dian-Yu Lin: collection and assembly of data, data interpretation, manuscript writing, and final approval of the manuscript. Min-Che Tung: data analysis and interpretation and final approval of the manuscript. Chin-Heng Lu: data analysis and interpretation, manuscript editing, manuscript revision, and final approval of manuscript. All authors contributed to the article and approved the submitted version.

\section{References}

[1] Birbrair A, Frenette PS. Niche heterogeneity in the bone marrow. Ann N Y Acad Sci. 2016;1370(1):82-96. https://doi.org/10.1111/nyas.13016.

[2] Bunnell BA, Flaat M, Gagliardi C, Patel B, Ripoll C. Adipose-derived stem cells: isolation, expansion and differentiation. Methods. 2008;45(2):115-20. https://doi.org/ 10.1016/j.ymeth.2008.03.006.

[3] Gregory CA, Prockop DJ, Spees JL. Non-hematopoietic bone marrow stem cells: molecular control of expansion and differentiation. Exp Cell Res. 2005;306(2):330-5. https://doi.org/10.1016/j.yexcr.2005.03.018.

[4] Huang GT, Gronthos S, Shi S. Mesenchymal stem cells derived from dental tissues vs. those from other sources: their biology and role in regenerative medicine. J Dent Res. 2009;88(9):792-806. https://doi.org/10.1177/0022034509340867.

[5] Weiss ML, Troyer DL. Stem cells in the umbilical cord. Stem Cell Rev. 2006;2(2): 155-62. https://doi.org/10.1007/s12015-006-0022-y.

[6] Raileanu VN, Whiteley J, Chow T, et al. Banking mesenchymal stromal cells from umbilical cord tissue: large sample size analysis reveals consistency between donors. Stem Cells Transl Med. 2019;8(10):1041-54. https://doi.org/10.1002/sctm.19-0022.

[7] Zuk PA, Zhu M, Ashjian P, et al. Human adipose tissue is a source of multipotent stem cells. Mol Biol Cell. 2002;13(12):4279-95. https://doi.org/10.1091/mbc.e02-020105.

[8] Mirzaeian L, Eftekhari-Yazdi P, Esfandiari F, et al. Induction of mouse peritoneum mesenchymal stem cells into germ cell-like cells using follicular fluid and cumulus cells-conditioned media. Stem Cells Dev. 2019;28(8):554-64. https://doi.org/10. 1089/scd.2018.0149.

[9] Shen J, Zheng J, Saxena R, Zhang C, Tang L. Novel source of human hematopoietic stem cells from peritoneal dialysis effluents. Stem Cell Res. 2015;15(2):299-304. https://doi.org/10.1016/j.scr.2015.07.003.

[10] Strioga M, Viswanathan S, Darinskas A, Slaby O, Michalek J. Same or not the same? Comparison of adipose tissue-derived versus bone marrow-derived mesenchymal stem and stromal cells. Stem Cells Dev. 2012;21(14):2724-52. https://doi.org/10. 1089/scd.2011.0722.

[11] Vezzani B, Shaw I, Lesme H, et al. Higher pericyte content and secretory activity of microfragmented human adipose tissue compared to enzymatically derived stromal vascular fraction. Stem Cells Transl Med. 2018;7(12):876-86. https://doi.org/10. 1002/sctm.18-0051.

[12] Dominici M, Le Blanc K, Mueller I, et al. Minimal criteria for defining multipotent mesenchymal stromal cells. The International Society for Cellular Therapy position statement. Cytotherapy. 2006;8(4):315-7. https://doi.org/10.1080/14653240600855905.

[13] Coronado RE, Somaraki-Cormier M, Ong JL, Halff GA. Hepatocyte-like cells derived from human amniotic epithelial, bone marrow, and adipose stromal cells display enhanced functionality when cultured on decellularized liver substrate. Stem Cell Res. 2019;38:101471. https://doi.org/10.1016/j.scr.2019.101471.

[14] McClusky 3rd DA, Mirilas P, Zoras O, Skandalakis PN, Skandalakis JE. Groin hernia: anatomical and surgical history [published correction appears in Arch Surg. 2006 Dec;141(12):1236]. Arch Surg. 2006;141(10):1035-42. https://doi.org/10.1001/ archsurg.141.10.1035.

[15] San-Marina S, Sharma A, Voss SG, Janus JR, Hamilton 3rd GS. Assessment of scaffolding properties for chondrogenic differentiation of adipose-derived mesenchymal stem cells in nasal reconstruction. JAMA Facial Plast Surg. 2017;19(2):108-14. https://doi.org/10.1001/jamafacial.2016.1200.

[16] Mahla RS. Stem cells applications in regenerative medicine and disease therapeutics. Int J Cell Biol. 2016;2016:6940283. https://doi.org/10.1155/2016/6940283.

[17] Yorukoglu AC, Kiter AE, Akkaya S, Satiroglu-Tufan NL, Tufan AC. A concise review on the use of mesenchymal stem cells in cell sheet-based tissue engineering with special emphasis on bone tissue regeneration. Stem Cells Int. 2017;2017:2374161. https://doi.org/10.1155/2017/2374161.

[18] Chu DT, Nguyen Thi Phuong T, Tien NLB, et al. Adipose tissue stem cells for therapy: an update on the progress of isolation, culture, storage, and clinical application. J Clin Med. 2019;8(7):917. Published 2019 Jun 26. doi: https://doi.org/10.3390/jcm8070917

[19] Köckerling F, Simons MP. Current concepts of inguinal hernia repair. Visc Med. 2018; 34(2):145-50. https://doi.org/10.1159/000487278.

[20] Schneider S, Unger M, van Griensven M, Balmayor ER. Adipose-derived mesenchymal stem cells from liposuction and resected fat are feasible sources for regenerative medicine. Eur J Med Res. 2017;22(1):17. Published 2017 May 19. doi: https://doi. org/10.1186/s40001-017-0258-9

[21] Ankrum JA, Ong JF, Karp JM. Mesenchymal stem cells: immune evasive, not immune privileged. Nat Biotechnol. 2014;32(3):252-60. https://doi.org/10.1038/nbt.2816.

[22] Fraser JK, Wulur I, Alfonso Z, Hedrick MH. Fat tissue: an underappreciated source of stem cells for biotechnology. Trends Biotechnol. 2006;24(4):150-4. https://doi.org/ 10.1016/j.tibtech.2006.01.010.

[23] Stolzing A, Jones E, McGonagle D, Scutt A. Age-related changes in human bone marrow-derived mesenchymal stem cells: consequences for cell therapies. Mech Ageing Dev. 2008;129(3):163-73. https://doi.org/10.1016/j.mad.2007.12.002.

[24] M J Oedayrajsingh-Varma 1, S M van Ham, M Knippenberg, M N Helder, J KleinNulend, T E Schouten, M J P F Ritt, F J van Millige. Adipose tissue-derived mesenchymal stem cell yield and growth characteristics are affected by the tissue-harvesting procedure. Cytotherapy 2006;8(2):166-77. doi:https://doi.org/10.1080/14653240 6006211PMID: 16698690 DOI: https://doi.org/10.1080/14653240600621125 